\title{
Suppression of Shastry-Sutherland phase driven by electronic concentration reduction in magnetically frustrated $\mathrm{Ce}_{2} \mathbf{P d}_{2} \mathbf{S n}_{1-y} \mathrm{In}_{y}$ alloys
}

\author{
J.G. Sereni ${ }^{1}$, J. Roberts ${ }^{2}$, F. Gastaldo ${ }^{2}$, M. Giovannini ${ }^{2}$ \\ ${ }^{1}$ Low Temperature Division, CAB-CNEA and CONICET, 8400 San Carlos de Bariloche, Argentina \\ ${ }^{2}$ Dipartimento di Chimica, Universita' di Genova, Via Dodecaneso 33, I-16146 Genova, Italy
}

(Dated: February 13, 2019)

\begin{abstract}
Shastry-Sutherland lattice was observed as alternative ground state in Rare Earth intermetallic with $\mathrm{Mo}_{2} \mathrm{~B}_{2} \mathrm{Fe}$ and $\mathrm{U}_{2} \mathrm{Pt}_{2} \mathrm{Sn}$ anisotropic structures where magnetic frustration is favored. In the case of $\mathrm{Ce}_{2} \mathrm{Pd}_{2} \mathrm{Sn}$, it was shown that such phase can be suppressed by the application of magnetic field and, in this work, its stability is studied as a function of the electronic concentration by doping the $\operatorname{Sn}(4+)$ lattice with $\operatorname{In}(3+)$ atoms. Magnetic and specific heat measurements show that around $50 \%$ substitution the Shastry Sutherland lattice vanishes in a critical point. This result confirms the strong dependence of that phase on the electron density because a recent investigation on the $\mathrm{Pd}$ rich solid solution $\mathrm{Ce}_{2+\epsilon} \mathrm{Pd}_{2-\epsilon} \operatorname{In}_{1-x} \mathrm{Sn}_{x}$ (with $\epsilon<0$ ) demonstrates that atomic disorder dominates the phase diagram at intermediate $\mathrm{Sn} / \mathrm{In}$ concentration inhibiting magnetic frustration effects. In the alloys investigated in this work, the $\epsilon>0$ character stabilizes the ferromagnetic ground state all along the concentration, allowing the Shastry Sutherland lattice formation on the Sn rich side.
\end{abstract}

\section{INTRODUCTION}

Intermetallic compounds with the formula $R_{2} T_{2} X$ (with $R=$ rare earths, $T=$ transition metals and $X=$ $\mathrm{Sn}, \mathrm{In}, \mathrm{Pb}$ and $\mathrm{Cd}$ ) have received considerable attention due to their varied behaviors, such as intermediate valence [1], non-Fermi liquid [2, 3] and quantum phase transitions 3, 4. Most of these compounds crystallize in the tetragonal $\mathrm{Mo}_{2} \mathrm{FeB}_{2}$-type [5], whereas a few of them are formed in a related variant $\mathrm{U}_{2} \mathrm{Pt}_{2} \mathrm{Sn}$ type. Both structures consist of alternate $R$-planes and $T / X$ planes stacked along the $c$ axis. In the first layers, magnetic $R$ atoms form a network of triangles and squares (mimicking a pinwheel mosaic). This geometry provides the ideal conditions for magnetic frustration and the consequent formation of the ShastrySutherland phase as alternative ground state, provided that the interactions between next neighbors may form a square lattice of magnetic dimers. In fact, ShastrySutherland $(\mathrm{ShSu})$ lattices [6] were observed in intermetallic compounds like $\mathrm{Yb}_{2} \mathrm{Pt}_{2} \mathrm{~Pb}$ [7, 8, crystallizing in the $\mathrm{U}_{2} \mathrm{Pt}_{2} \mathrm{Sn}$-type and $\mathrm{Ce}_{2} \mathrm{Pd}_{2} \mathrm{Sn}\left[9\right.$ with the $\mathrm{Mo}_{2} \mathrm{FeB}_{2}$ type.

In the case of $\mathrm{Ce}_{2} \mathrm{Pd}_{2} \mathrm{Sn}$ the $\mathrm{ShSu}$ phase is detected between a temperature $\left(T_{S}\right)$ at which neighboring Ce atoms form magnetic dimers distributed at the corners of a simple square 2D lattice and a FM transition $\left(T_{C}\right)$ at which inter-plane interactions transform the system into a $3 \mathrm{D}$ magnet [9]. The magnetic stability of this phase in $\mathrm{Ce}_{2} \mathrm{Pd}_{2} \mathrm{Sn}$ was previously studied under magnetic field [10, detecting that it is suppressed at a critical field $B_{c r}=0.11 \pm 0.01 \mathrm{~T}$.

In order to recognize the stability of that phase as a function of electronic concentration, the progressive substitution of tetravalent $(4+)$ Sn atoms by trivalent $(3+)$ In ones was investigated. The isotypic $\mathrm{Ce}_{2} \mathrm{Pd}_{2} \mathrm{In}$ is known to form in the same $\mathrm{Mo}_{2} \mathrm{~B}_{2} \mathrm{Fe}$-type structure [5]. However, instead of a stoichiometric compound it was determined that it forms as a solid solution $\mathrm{Ce}_{2+\epsilon} \mathrm{Pd}_{2-\epsilon} \mathrm{In}$ [11] around that concentration. Moreover, the low temperature magnetic properties were recognized to depend on the relative excess of Ce $(\epsilon>0)$ or $\mathrm{Pd}(\epsilon<0)$ resulting in ferromagnetic $(\mathrm{FM})$ or antiferromagnetic (AFM) ground states (GS) respectively [11, 12. These two branches on the In rich side allows to study the ShSu lattice stability within the $\mathrm{Ce}_{2} \mathrm{Pd}_{2} \mathrm{Sn}_{1-y} \operatorname{In}_{y}$ alloys following to comparable trails, one pointing to a FM and the other to an AFM final GS.

Recently, the $\epsilon<0$ branch, i.e. starting from the AFM sate in $\mathrm{Ce}_{2} \mathrm{Pd}_{2}\left(\mathrm{In}_{1-x} \mathrm{Sn}_{x}\right)$, was reported [13] to show relevant atomic disorder for $x \geq 0.4$ that inhibits the formation of the ShSu lattice at higher Sn content. Such magnetic disorder is reflected in a broadening of the $T_{N}(x)$ transition at that concentration. Complementary, in this work we report on the stability of the ShSu phase following the rich Ce, $\mathrm{Ce}_{2.15} \mathrm{Pd}_{1.95} \mathrm{Sn}_{1-y} \mathrm{In}_{y}$ FM branch, i. e. with $\epsilon>0$, that forms continuously along the full $\mathrm{Sn} / \mathrm{In}$ composition and show well defined magnetic transitions.

\section{EXPERIMENTAL DETAILS AND RESULTS}

\section{A. Sample Preparation and Characterization}

The samples were prepared by weighing the proper amounts of elements, then arc melted in an Argon atmosphere on a water cooled copper hearth with a tungsten electrode. To ensure good homogeneity the buttons were turned over and remelted several times. Weight losses after melting were always smaller than 0.5 mass 
percent. Later, the samples were annealed at $750^{\circ} \mathrm{C}$ for ten days and quenched in cold water. Scanning electron microscopy (SEM), and electron probe micro-analysis (EPMA) based on energy-dispersive X-ray spectroscopy were used to examine phase compositions. The compositional contrast was revealed in unetched samples by means of a backscattered electron detector (BSE). The composition values derived were usually accurate to 1 at\%. X-ray diffraction (XRD) was performed on powder samples using a vertical diffractometer X-Pert with $\mathrm{CuK} \mathrm{K}_{\alpha}$ radiation. Lattice parameters slightly change along the concentration range from $a=7.765 \AA$ and $c=3.902 \AA$, with a $c / a=0.5026$ ratio of the $x=0$ mother compound [9].

DC-magnetization measurements were carried out using a standard SQUID magnetometer operating between 1.8 and $320 \mathrm{~K}$, and as a function of field up to $5 \mathrm{~T}$. Specific heat was measured using a standard heat pulse technique in a semi-adiabatic He-3 calorimeter in the range between 0.5 and $20 \mathrm{~K}$, at zero applied magnetic field.

\section{B. Magnetic Properties}

High temperature $(T>30 \mathrm{~K})$ magnetic susceptibility results are properly described by a $\chi=\chi_{c w}+\chi_{p}$ dependence, see Fig. 1a, where the first term corresponds to the temperature dependent Curie-Weiss contribution $\chi_{c w}=\frac{C c}{T+\theta}$ and the second to a Pauli-like contribution. This weak $\chi_{p}$ contribution is observed along the full concentration range with values ranging within $\left.\chi_{p}=0.6 \pm 0.4\right) 10^{-3} \mathrm{emu} /$ Ce at.Oe. From the inverse of $\chi_{c w}$ one may extract the Curie constant $(\mathrm{Cc})$ which indicates a large but not fully developed $\mathrm{Ce}^{3+}$ magnetic moment (i.e. $\mu_{e f f}=2.32 \mu_{B}$ per Ce atom). The paramagnetic temperature extrapolated from high temperature $(T>50 \mathrm{~K})$ is notably weak $\theta_{P} \leq 7 \mathrm{~K}$ all along the concentration variation, excluding any relevant effect of local moments-conduction band hybridization or Kondo effect.

On the stoichiometric $S n$ limit, the six fold degenerated state established by Hund's rules for the $J=5 / 2$ angular momentum of Ce atoms is split by the crystal electric field (CEF) effect into three Kamer's doublets with the excited ones at $\Delta_{1} \approx 65 \mathrm{~K}$ and $\Delta_{1} \approx 230 \mathrm{~K}[9$. Similar value $\left(\Delta_{1} \approx 60 \mathrm{~K}\right)$ was reported for the first excited doublet on the In side 12. This coincidence indicates that no relevant modification of the CEF occurs along the Sn/In substitution guaranteeing that the low temperature magnetic properties is governed by a pure doublet GS.

Magnetization $M(B)$ curves at $T=1.8 \mathrm{~K}$ are shown in the inset of Fig. 1 1 . The saturation values slightly increases from $1.02 \mu_{B}$ to $1.13 \mu_{B}$ between pure $S n$ and

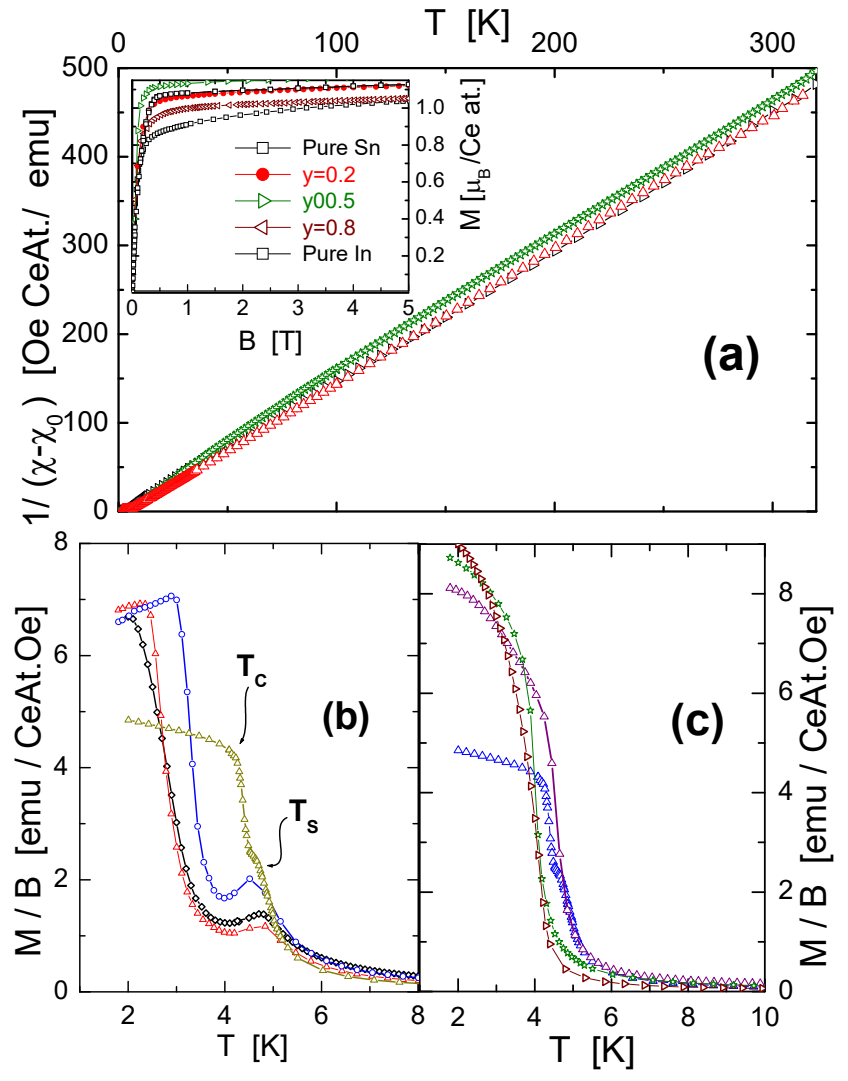

FIG. 1. (a) Inverse magnetic susceptibility from $1.8 \mathrm{~K}$ up to room temperature measured in a field $B=1 \mathrm{~T}$. Inset: magnetization versus field up to $5 \mathrm{~T}$, measured at $1.8 \mathrm{~K}$. (b) Low temperature susceptibility of $\mathrm{Sn}$ rich alloys, measured with $B=100 \mathrm{Oe}$, showing how the $\mathrm{ShSu}$ phase is progressively suppressed by increasing In content. (c) The same for the In rich concentrations.

In limits, with a maximum value at the critical concentration $y_{c r}=0.5$ of $1.18 \mu_{B}$.

The low temperature magnetization normalized by field $(M / B)$ is presented in Fig. 1p including the $0 \leq$ $y \leq 0.5$ samples where both $T_{S}(y)$ and $T_{C}(y)$ transitions are clearly identified. Fig. 11 contains the FM magnetization of the $0.5 \leq y \leq 1$ samples.

\section{Specific Heat}

To extract the magnetic contribution $\left(C_{m}\right)$ to the measured specific heat $\left(C_{P}\right)$, the $\mathrm{La}_{2} \mathrm{Pd}_{2} \mathrm{In}$ compound was used as a reference for the phonon subtraction 9] as $C_{m}=C_{P}(T)-C\left(L a_{2} P d_{2} I n\right)$. On the $S n$-rich side, the two magnetic transitions observed in magnetic measurements (see Fig. 1b) are reflected in corresponding $C_{m}(T) / T$ jumps presented in Fig. 2 On the $S n$-rich 


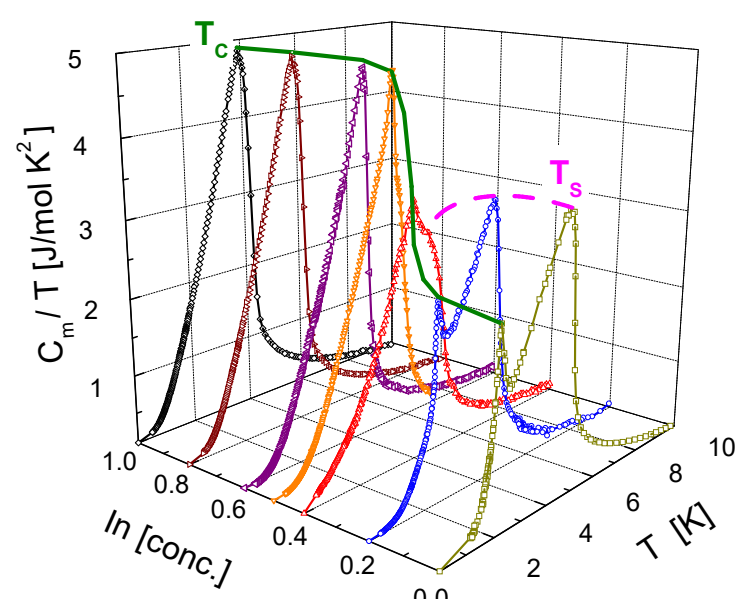

FIG. 2. Magnetic contribution to the specific heat of the measured alloys in a $3 \mathrm{D}$ representation. $\mathrm{T}_{C}(y)$ indicates the FM transition (continuous curve) and $T_{S}(y<5)$ the transition into the Shastry Sutherland phase (dashed curve)

side, the specific heat jump at the $T=T_{S}$ (dashed curve) transition between paramagnetic and ShSu phase exhibits a second order character, whereas the lower one $\left(T_{C}\right)$ associated to the ferromagnetic phase shows the characteristic of a first order. However, above the critical concentration $\left(y_{c r} \approx 0.5\right)$, at which the ShSu phase is suppressed, the only transition $T_{C}$ becomes of second order keeping the tail originated in magnetic fluctuations arising above the transition.

Coincidentally with the previous study on stoichiometric $\mathrm{Ce}_{2} \mathrm{Pd}_{2} \mathrm{Sn}$, the ShSu phase suppression by magnetic field, $T_{S}(y)$ slightly changes in temperature with In concentration $T_{S}(0 \leq y \leq 0.5)=(4.5 \pm 0.2 \mathrm{~K})$ whereas $T_{C}(y=0)=2.1 \mathrm{~K}$ increases from up to reach the critical point at $T_{c r}\left(y_{c r}\right)=4.15 \mathrm{~K}$.

Notably, the height of $C_{m} / T$ at $T_{C}(0 \leq y<0.5)$ is clearly larger than for $T_{S}(0.5 \leq y \leq 1)$. This indicates that the transition at $T=T_{S}$ corresponds to an intermediate phase that involves a partial condensation of the available degrees of freedom which are then condensed into the FM phase at $T<T_{C}$. On the contrary, the transition at for $x \geq y_{c r}$ involves all magnetic degrees of freedom.

\section{DISCUSSION}

\section{A. Magnetic phase diagram}

Based on magnetic and specific heat results, the evolution of the Shastry-Sutherland $\left(T_{S}\right)$ and Curie $\left(T_{C}\right)$ transitions can be traced as a function of In increase

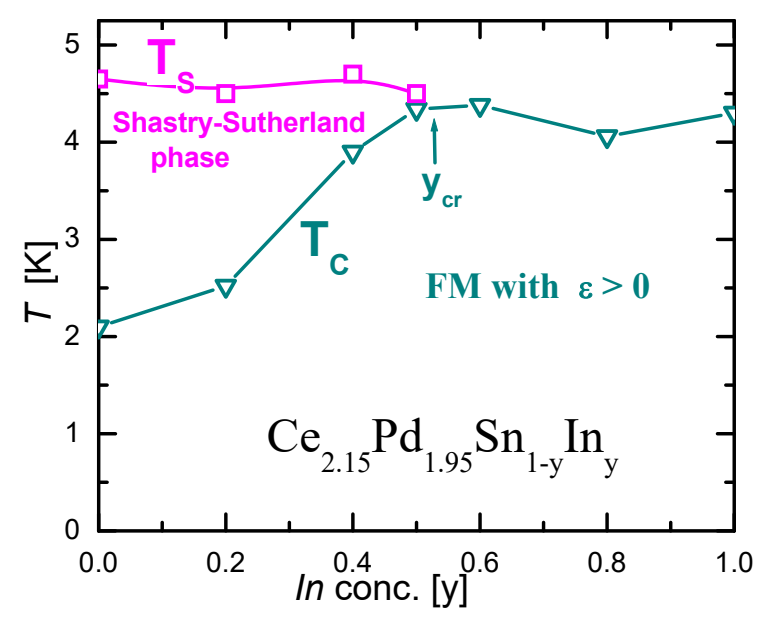

FIG. 3. Magnetic phase diagram showing the evolution of the Curie $\left(T_{C}\right)$ and Shastry-Sutherland $\left(T_{S}\right)$ transitions as a function of In increase.

(upper FM curves). One can see that the ShastrySutherland holds up to about $50 \%$ of $I n$ substitution.

On the $\mathrm{Sn}$-rich side, the characteristics of the $\mathrm{ShSu}$ phase recognized in the $\mathrm{Ce}_{2} \mathrm{Pd}_{2} \mathrm{Sn}$ compound [9] can be traced up to the sample with $40 \%$ of In concentration. Although the difference between $T_{S}$ and $T_{C}$ in the specific heat measurements is observed as a structure in a unique anomaly, the $M(T)$ dependence allows to better distinguish both transitions at that concentration, see Fig,4a. Both properties are better compared in the lower panel (Fig $4 \mathrm{~b}$ ), using as magnetic parameter the second derivative because in FM systems the internal magnetic energy $U_{m} \propto M^{2}$ and therefore $C_{m} \propto \delta M^{2} / \delta^{2} T$.

Another identification of the ShSu phase can be done through its suppression by a magnetic field, which occurs at $B_{c r}(y=0.4) \approx 500$ Oe, see the inset in Fig 4 a. This behavior can be compared with the suppression of the ShSu phase of the stoichiometric compounds $\mathrm{Ce}$ ${ }_{2} \mathrm{Pd}_{2} \mathrm{Sn}$ [14, taking into account that $B_{c r}(y=0.4) \approx$ $1 / 2 B_{c r}(y=0)$.

\section{B. Critical point}

The full suppression of the ShSu phase by increasing In concentration is observed slightly above the $50 \%$ of substitution, where the $T_{S}(y)$ transition joints $T_{C}(y)$ FM phase boundary. In Fig 5 the specific heat transitions of two neighboring concentrations, $y=0.5$ and $y=0.6$, are compared making evident that the later does not show any structure around its maximum which is still detected in the $x=0.5$ one. Coincidentally, there is practically no difference between respective entropies 


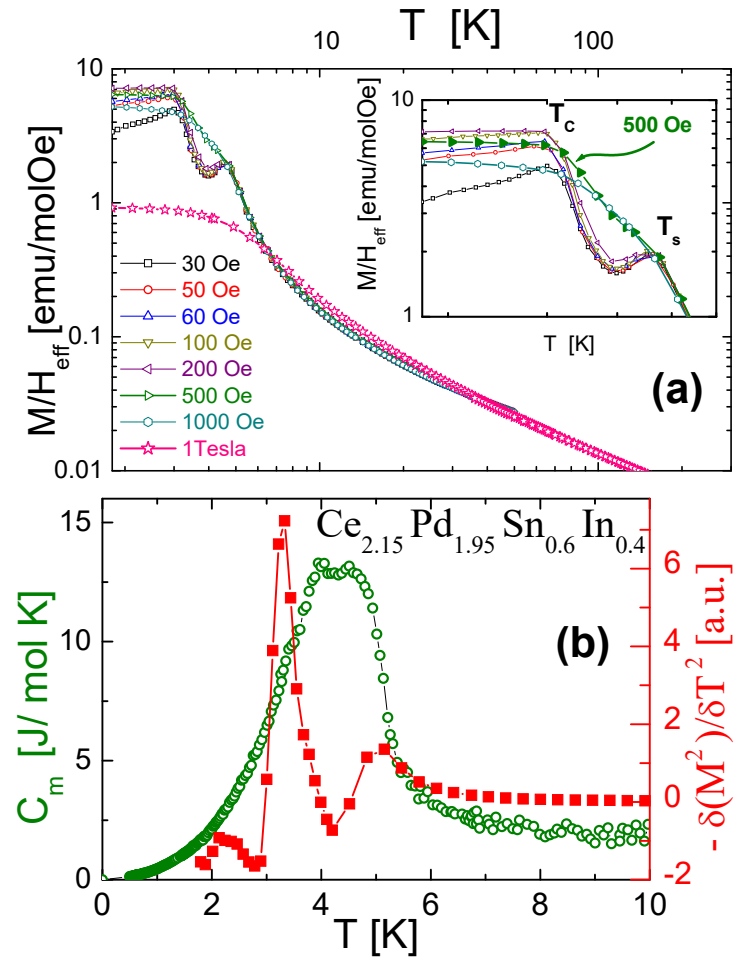

FIG. 4. (a) Progressive Shastry Sutherland phase suppression by magnetic field at $40 \%$ In in a zero field cooling process. Inset: detail of the mentioned suppression. (b) Comparison between specific heat measurement and magnetization derivative.

collected up to the transition.

In the inset of Fig 5 the same comparison is performed on respective magnetization derivatives making the difference more evident. Notably, the first order type cusp in the $y=0.5$ sample fully disappears at the following concentration $y=0.6$.

\section{Physical behavior beyond the critical point}

Between $y=0.6$ and 1, the typical FM $M(T)$ dependence is observed, see Fig 1 ; , while $C_{m} / T$ shows the same behavior like for pure In. Independently of these low temperature features, sample $x=0.8$ shows an unexpected ferromagnetic signal in the thermal dependence of its magnetic susceptibility arising at $T \leq 30 \mathrm{~K}$, see Fig.6. This FM contribution was analyzed as a function of magnetic field at $T=25 \mathrm{~K}$ reflected in an increase of the measured magnetization with field $\Delta M_{25 K}(B)$, see the inset in Fig 6. This FM signal is found to saturate at around $0.3 \mathrm{~T}$. Such contribution cannot be explained by any foreign phase constituted by any combination of the four elements Ce-Pd-Sn-In,

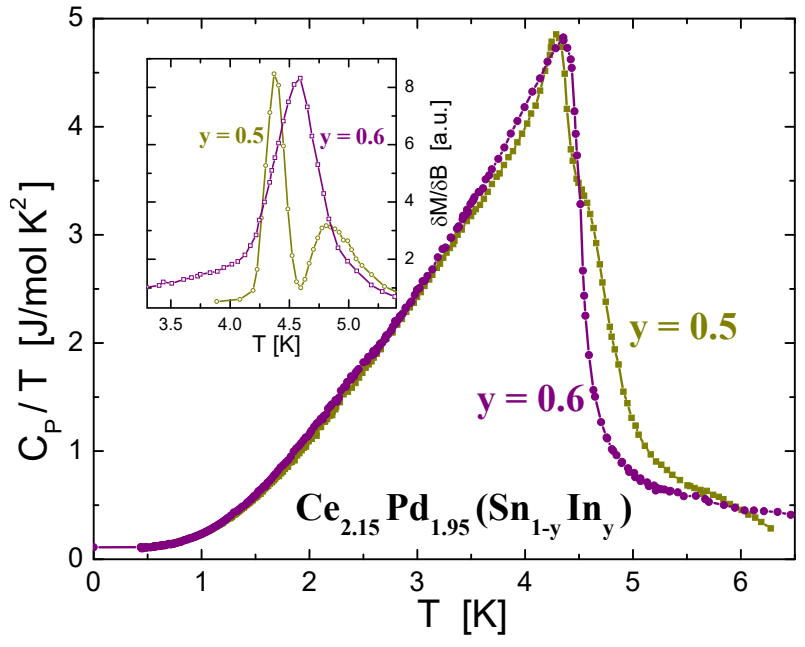

FIG. 5. Critical concentration determined by specific heat and, in the inset, by the magnetization derivatives of samples $y=0.5$ and $y=0.6$.

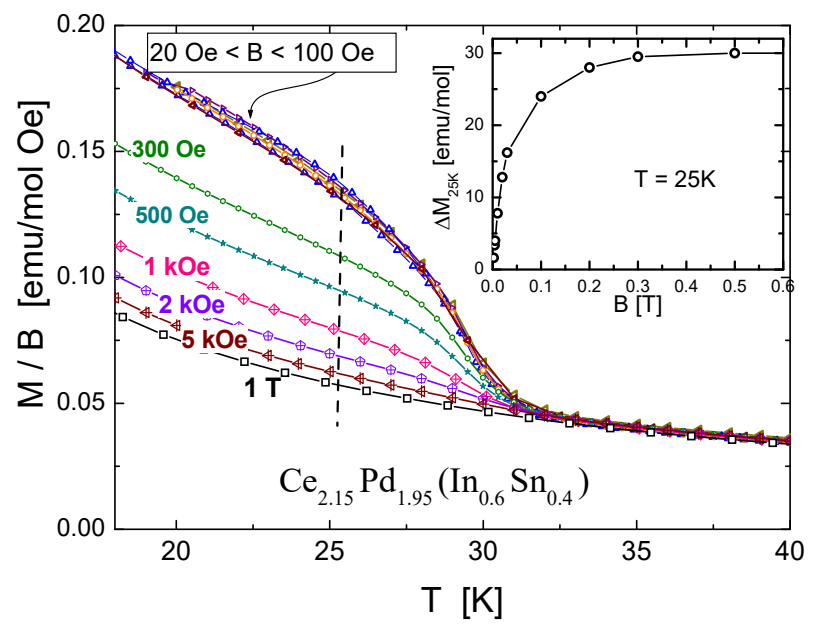

FIG. 6. Field dependence of the unexpected ferromagnetic contribution below about $30 \mathrm{~K}$ tentatively attributed to spin density waves formation. Inset: Magnetization increase upon the bulk magnetization at $25 \mathrm{~K}$.

therefore it could be more likely related to the onset of spin density waves. A microscopic investigation on the nature of this anomaly is required to confirm its origin.

\section{CONCLUSIONS}

This study demonstrates that, contrary to the AFM ( $\epsilon<0)$ branch of $\mathrm{Ce}_{2+\epsilon} \mathrm{Pd}_{2-\epsilon}\left(\operatorname{In}_{1-x} \mathrm{Sn}_{x}\right)$ alloys, the FM $(\epsilon>0)$ one shows well defined transitions with irrele- 
vant atomic disorder produced by $\mathrm{Sn} / \mathrm{In}$ substitution. As a consequence the upper $\left(T=T_{S}\right)$ and the lower $\left(T=T_{C}\right)$ boundaries of the ShSu phase can be clearly traced up to $y \approx 50 \%$ of $\mathrm{Sn} / \mathrm{In}$ substitution. The reduction of the electronic concentration driven by doping the $\operatorname{Sn}(4+)$ lattice with $\operatorname{In}(3+)$ atoms progressively weakens the stability of that phase reflected in the monotonous growing of the FM phase in detriment of the former and the consequent increase of $T_{C}(y)$. Magnetic field effect, analyzed at the edge of the critical concentration $y=0.4$, shows that only $1 / 2$ of field is required to sup- press the $\mathrm{ShSu}$ phase in comparison to stoichiometric $\mathrm{Ce}_{2} \mathrm{Pd}_{2} \mathrm{Sn}$.

Although Ce rich content $(\epsilon>0)$ reduces atomic disorder effects allowing to extend the $\mathrm{ShSu}$ phase range, further decrease of electronic concentration after $\mathrm{Sn} / \mathrm{In}$ substitution definitively suppresses the possibility of dimers formation on which simple square $\mathrm{ShSu}$ lattice builds up. Since this lattice forms within the Ce planes, one may expect that on the $I n$-rich side (i.e. $y>0.5$ ) interplane interactions arise making the system to behave like a 3D - FM as soon as the magnetic order parameter arises.
[1] D. Kaczorowski, P. Rogl, K. Hiebl, in Magnetic behavior in a series of cerium ternary intermetallics: $C_{2} T_{2}$ In ( $T=N i, C u, R h, P d, P t$, and $A u)$; Phys. Rev. B 54 (1996) 9891.

[2] S.K. Dhar, R. Settai, Y. nuki, A. Galatanu, Y. Haga, P. Manfrinetti, M. Pani, in Synthesis, crystal structure and magnetic properties of $Y b_{8} A g_{18.5} A l_{47.5}, Y b_{2} P d_{2} C d$ and $Y b_{1.35} P d_{2} C d_{0.65}$; J. Magn. Magn. Materials 308 (2007) 143.

[3] E. Bauer, G. Hilscher, H. Michor, C. Paul, Y. Aoki, H. Sato, D. Adroja, J.-G. Park, P. Bonville, C. Godart, J. Sereni, M. Giovannini, and A. Saccone, J. Phys.: Condens. Matter 17, S999 (2005).

[4] T. Muramatsu, T. Kanemasa, T. Kagayama, K. Shimizu, Y. Aoki, H. Sato, M. Giovannini, P. Bonville, V. Zlatic,T. Aviani, R. Khasanov, C. Rusu, A. Amato, K. Mydeen, K. Nicklas, H. Michor, E. Bauer, in Reentrant quantum criticality in $\mathrm{Yb}_{2} \mathrm{Pd}_{2} \mathrm{Sn}$; Phys. Rev. B 83 (2011) 18.

[5] F. Fourgeot, P. Gravereau, B. Chevalier, L. Fourns, J. Etourneau, in Effect of the chemical composition on the structural properties of the antiferromagnetic ternary stannides $\mathrm{Ce}_{2} P d_{2+x} \mathrm{Sn}_{1 x}(0.04(3) \leq x \leq 0.21(4))$; J. Alloys Comp. 238 (1996) 102.

[6] B. S. Shastry and B. Sutherland, Physica 108B (1981) 1069.

[7] M.S. Kim and M.C. Aronson, in Heavy fermion compounds on the geometrically frustrated ShastrySutherland lattice; J. Phys.: Condens. Matter 23 (2011) 164204.

[8] L.S. Wu, W.J. Gannon, I.A. Zaliznyak, A.M. Tsvelik,
M. Brockmann, J.-S. Caux, M.S. Kim, Y. Qiu, J.R.D. Copley, G. Ehlers, A. Podlesnyak, M. C. Aronson; in Orbital-exchange and fractional quantum number excitations in an f-electron metal, $\mathrm{Yb}_{2} \mathrm{Pt}_{2} \mathrm{~Pb}$; Science 352 (2016) 1206.

[9] J. G. Sereni, M. G-Berisso, A. Braghta, G. Schmerber, J. P. Kappler; Unstable Shastry-Sutherland phase in $\mathrm{Ce}_{2} \mathrm{Pd}_{2} \mathrm{Sn}$, Phys. Rev. 80 (2009) 024428.

[10] J. G. Sereni, M. Gomez Berisso, G. Schmerber, J. P. Kappler; Suppression of the Shastry-Sutherland phase in $\mathrm{Ce}_{2} \mathrm{Pd}_{2} \mathrm{Sn}$ at a field-induced critical point, Phys. Rev. B 81 (2010) 184429.

[11] M. Giovannini, H. Michor, E. Bauer, and G. Hilscher, P. Rogl, T. Bonelli, F. Fauth, P. Fischer, T. Herrmannsdo, L. Keller, W. Sikora, A. Saccone and R. Ferro; in Effect of nonstoichiometry on the transition from ferromagnetism to antiferromagnetism in the ternary indides $C e_{1.95} P d_{2+2 x} I n_{1-x}$ and $C e_{2+x} P d_{1.85} \operatorname{In}_{1-x}$, Phys. Rev. B 61 (2000) 4044.

[12] J.G. Sereni, M. Giovannini, M. Gomez Berisso, A. Saccone; in Electron concentration effects on the ShastrySutherland phase stability in $\mathrm{Ce}_{2 x} \mathrm{Pd}_{2+y} \mathrm{In}_{1 z}$ solid solutions; Phys. Rev.B 83 (2011) 064419.

[13] J.G. Sereni, J. Roberts, F. Gastaldo, M. G-Berisso, M. Giovannin; Shastry-Sutherland phase formation in magnetically frustrated $C e_{2} P d_{2} I_{1-x} S n_{x}$ alloys. Materials Today: Proceedings (Elsevier), 2018, in presss.

[14] M. G- Berisso, J. G. Sereni, A. Braghta, G. Schmerber, B. Chevalier, J.P. Kappler, Field suppression of the modulated phase of $\mathrm{Ce}_{2} \mathrm{Pd}_{2} \mathrm{Sn}$; Physica B 404 (2009) 2930 . 